\title{
Sexual Dysfunction among Filipino Breast Cancer Patients
}

\author{
Arthur Gregory A. Lui, ${ }^{1}$ Joanne Marie L. Balbuena, ${ }^{1}$ \\ Antonio L. Faltado Jr., ${ }^{2}$ Charles Vincent O. $\mathrm{Uy}^{2}$ and Heinrik Martin Jude S. Strebel ${ }^{1}$ \\ ${ }^{1}$ Section of Medical Oncology, Department of Medicine, College of Medicine and Philippine General Hospital, University of the Philippines Manila \\ 2Department of Medicine, College of Medicine and Philippine General Hospital, University of the Philippines Manila
}

\begin{abstract}
Introduction. Sexual function is an important aspect of quality of life, and can be drastically affected in ill patients. Very few studies (and apparently none among Filipinas) looked into sexual dysfunction among females with breast cancer $(\mathrm{BrCa})$; prevalence also is not well defined. This study evaluates the prevalence of sexual dysfunction among Filipino patients with $\mathrm{BrCa}$, and assesses which treatment or if duration of illness, age, BMI, smoking history, diabetes, hypertension significantly contributed to the dysfunction.
\end{abstract}

Methods. A cross sectional study was conducted among $\mathrm{BrCa}$ patients consulting at the outpatient medical oncology clinic of a government tertiary hospital. Study population included those diagnosed and was with breast cancer over a 3-months period, with a calculated sample size of 60 (within $81 \pm 10 \%$ prevalence rate, Cl 95\%). A validated translated version of the Female Sexual Function Index (FSFI) 19-item questionnaire that looked into 6 domains (arousal, lubrication, desire, pain, orgasm, and satisfaction) was used. Sexual dysfunction was defined as an FSFI score of $<26.55$.

Results. Of the 97 respondents, mean age was 49.4 years old and mean BMI of 24.8 . About $78 \%$ received chemotherapy, $26 \%$ hormonal therapy. $15 \%$ radiotherapy, $82 \%$ modified radical mastectomy (MRM), and $71 \%$ received both MRM and chemotherapy at the time of interview. Duration of cancer was $\geq 6$ months in $72 \%$ of subjects. There were $97.9 \%$ who had sexual dysfunction which is similar to prevalence rates (64-98\%) in other studies. Age, BMI, smoking history, hypertension, diabetes mellitus, chemotherapy, surgery, hormonal therapy, radiation therapy, and duration of illness were shown not to be significant predictors of sexual dysfunction among Filipinas with BrCa by bivariate analysis.

Presented and won 2nd place at the Philippine Society of Oncology 2014 Annual Convention; presented and a finalist at the Philippine Society of Medical Oncology 2014 Annual Convention.

Corresponding author: Heinrik Martin Jude S. Strebel, MD, MCM(MO) Section of Medical Oncology

Department of Medicine

Philippine General Hospital

University of the Philippines Manila

Taft Avenue, Ermita, Manila 1000 Philippines

Tele/Fax: +632 5263775

Email: arik_strebel@yahoo.com
Conclusion. Sexual dysfunction is highly prevalent among female Filipino BrCa patients. Knowing such high prevalence should prompt health care providers to include interventions to improve quality of life of $\mathrm{BrCa}$ patients, including their sexual life.

Key Words: sexual dysfunction, breast cancer, Filipino

\section{Introduction}

To date, breast cancer is still the leading cause of cancer among Filipino women, accounting for $28 \%$ of the total cases. ${ }^{1}$ As treatment modalities improve for breast cancer, so is survival from this disease. And as patients live longer with this diagnosis, quality of life is an important aspect in the holistic management of breast cancer patients. One aspect of quality of life is sexual function, which is a basic need by Maslow's Hierarchy of needs. ${ }^{2}$ Discussion of sexual function is often overlooked among clinicians; and most patients fail to volunteer such information.

Sexual dysfunction is defined as a disturbance in the sexual response cycle or as pain with sexual intercourse. ${ }^{2}$ It can be symptomatic from biological problems or intrapsychic or interpersonal (psychogenic) conflicts or a combination of these factors. Female sexual dysfunction has traditionally included disorders of desire, arousal, pain, and inhibited orgasm. While epidemiologic data are limited, the available estimates in the general population are that $43 \%$ of women complain of at least one sexual problem, while 11$33 \%$ of survey and clinical samples fall within a specific problem category. ${ }^{3}$

Physiologically, breast cancer is likely to have a negative impact on sexual function, due to the toxic effect of chemotherapy on ovarian follicles and the ovarian ablation or bilateral oophorectomy done in some cases, which interfere with the production of female reproductive hormones. Psychologically, the breast is often referred to as the body part that is most strongly associated with women's femininity, maternal role and sexuality. Changes in the breast may not necessarily interfere with women's physical ability to have sexual intercourse; however, it is strongly associated with sexual well-being, body image and feminine identification.

The prevalence of sexual dysfunction among breast cancer patients has been investigated elsewhere. Prevalence rates of sexual dysfunction of breast cancer patients in some 
studies reviewed ranged from $64-98 \%$ (Netherlands 4 $~ 64 \%$; Australia $^{5}$ 70\%; France $27 \%$; Italy ${ }^{6} 96 \%$; Japan $798 \%$ ).

The Female Sexual Function Index (FSFI) ${ }^{3}$ is one of the questionnaires most commonly used in the assessment of sexual function in women. It is a validated test, which consists of 19 questions on the sexual activity performed in the last four weeks. It enables assessment of six sexual functioning domains: desire, arousal, lubrication, orgasm, satisfaction, and discomfort/pain. Sub-domains are scored considering the values of each question and its respective conversion factors and total FSFI Score is the sum of the six results, ranging from 2 to 36 and better levels of sexual function are indicated by highest scores.

Cavalheiro et al (Brazil) $)^{8}$ showed $43 \%$ reduction in FSFI score compared to control. Sbitti et al (Morocco) $)^{9}$ found that the most frequent sexual dysfunction were dyspareunia (65\%) followed by lubrication difficulties (54\%) and the absence or reduction of sexual desire $(48 \%$ and $64 \%$, respectively). About one third of subjects complained of inhibited female orgasm (40\%), lack of satisfaction $(37 \%)$, brevity of intercourse and arousal (38\%). The sexual dysfunctions were absent before diagnosis and management of breast cancer in $91.5 \%$ and of these $100 \%$ complained of a deterioration of the symptomatology after the various treatments.

Factors contributing to the observed variability in prevalence rates of sexual dysfunction are different across studies in patients' demographic and medical characteristics. A variety of demographic characteristics such as age and partner status are known to be associated with reports of sexual difficulties in the general population. Factors related to the disease itself (stage) and form of treatment (chemotherapy, type of surgery, radiotherapy and hormonal therapy) greatly affects the reported prevalence of sexual dysfunction. ${ }^{10-11}$

Data about sexual dysfunction in patients with breast cancer in the Philippines is lacking. This study is the first in the Philippines, allowing researchers to address diagnosis and treatment of sexual dysfunction among females with breast cancer. This study evaluates the prevalence and occurrence of sexual dysfunction among Filipinas with breast cancer.

\section{Methods}

This is a cross sectional study, which used validated Filipino questionnaire version of the FSFI. ${ }^{12-13}$ Female breast cancer patients from the outpatient oncology clinic of a government tertiary hospital, aged $19-65$ years with sexual activity within the last 4 weeks were included in the study. Those patients with neurologic diseases (i.e., spinal cord injury, multiple sclerosis), history of aorto-iliac surgery, pelvic trauma or irradiation, who failed to answer the questionnaire completely, who are currently pregnant, and who refused to participate were excluded from the study.
The University of the Philippines Manila Research Ethics Board (UPMREB) approved the protocol prior to data collection; data confidentiality was observed. If there were concerns regarding the presence of sexual dysfunction, the investigators advised the attending physician to refer the patient to Uro-Gynecology or Psychiatry Services for management.

The computed sample size was 60 , which was required to estimate the prevalence of sexual dysfunction within $81+$ $10 \%$, with a $95 \%$ confidence interval. Descriptive statistics such as mean $\pm \mathrm{SD}$ and range were computed for continuous data while frequency and percentages were presented for categorical data. To determine the association of sexual dysfunction with age, body mass index, smoking, alcohol consumption, duration of breast cancer, and presence of comorbidities, multiple logistic regression was used. A pvalue of $<0.05$ was considered significant. On the basis of sensitivity and specificity analyses from the original author of the FSFI, a total score of 26.55 was the optimal cut score for differentiating women with and without sexual dysfunction.

\section{Results}

Of the 97 respondents, mean age was 49.4 years and mean BMI of 24.8. About $78 \%$ received chemotherapy, $26 \%$ hormonal therapy, $15 \%$ radiotherapy, $82 \%$ modified radical mastectomy (MRM); $71 \%$ received both MRM and chemotherapy at the time of interview. Duration of cancer was $\geq 6$ months in $72 \%$ of subjects.

There were $97.9 \%$ Filipina breast cancer patients who had sexual dysfunction. Table 1 shows the mean and standard deviation FSFI scores based on the domain, with overall mean score of 11.72 , which is below the cut off score of 26.55. Table 2 shows the mean FSFI score by domain and by age group. Mean score for each domain of those $\leq 50$ years old were higher than those $\geq 50$ years old. Overall mean score was also higher in those $\leq 50$ years old.

Table 1. Mean FSFI Score Based on Domain ( $\mathrm{N}=97)$

\begin{tabular}{lc}
\hline \multicolumn{1}{c}{ Domain } & Mean Score, SD for All Ages \\
\hline Desire & $1.86,1.03$ \\
Arousal & $1.35,1.22$ \\
Lubrication & $1.63,1.74$ \\
Orgasm & $1.49,1.79$ \\
Satisfaction & $1.76,2.06$ \\
Pain and Discomfort & $3.63,2.29$ \\
Overall & $11.72,7.61$ \\
\hline
\end{tabular}

Age, BMI, smoking history, hypertension, diabetes mellitus, chemotherapy, surgery, hormonal therapy, radiation therapy, and duration of illness were shown not to be significant predictors of sexual dysfunction among Filipinas with BrCa by bivariate analysis (Table 3). Logistic regression was no longer performed due to negative results from bivariate analysis. 
Table 2. Mean FSFI Score by Domain and Age Group $(\mathrm{N}=97)$

\begin{tabular}{lcc}
\hline \multicolumn{1}{c}{ Domain } & $\begin{array}{c}\text { Mean Score, SD } \\
\text { for } \leq \mathbf{5 0} \text { Years Old }\end{array}$ & $\begin{array}{c}\text { Mean Score, SD } \\
\text { for }>\mathbf{5 0} \text { Years Old }\end{array}$ \\
\hline Desire & $2.09,1.00$ & $1.56,0.98$ \\
Arousal & $1.62,1.34$ & $0.97,0.94$ \\
Lubrication & $1.99,1.80$ & $1.13,1.53$ \\
Orgasm & $1.89,1.92$ & $0.96,1.44$ \\
Satisfaction & $2.14,2.16$ & $1.24,1.82$ \\
Pain and Discomfort & $3.77,2.10$ & $3.44,2.54$ \\
Overall & $13.50,7.94$ & $9.30,6.48$ \\
\hline
\end{tabular}

Table 3. Sexual Dysfunction by possible Related Risk Factors (N=97)

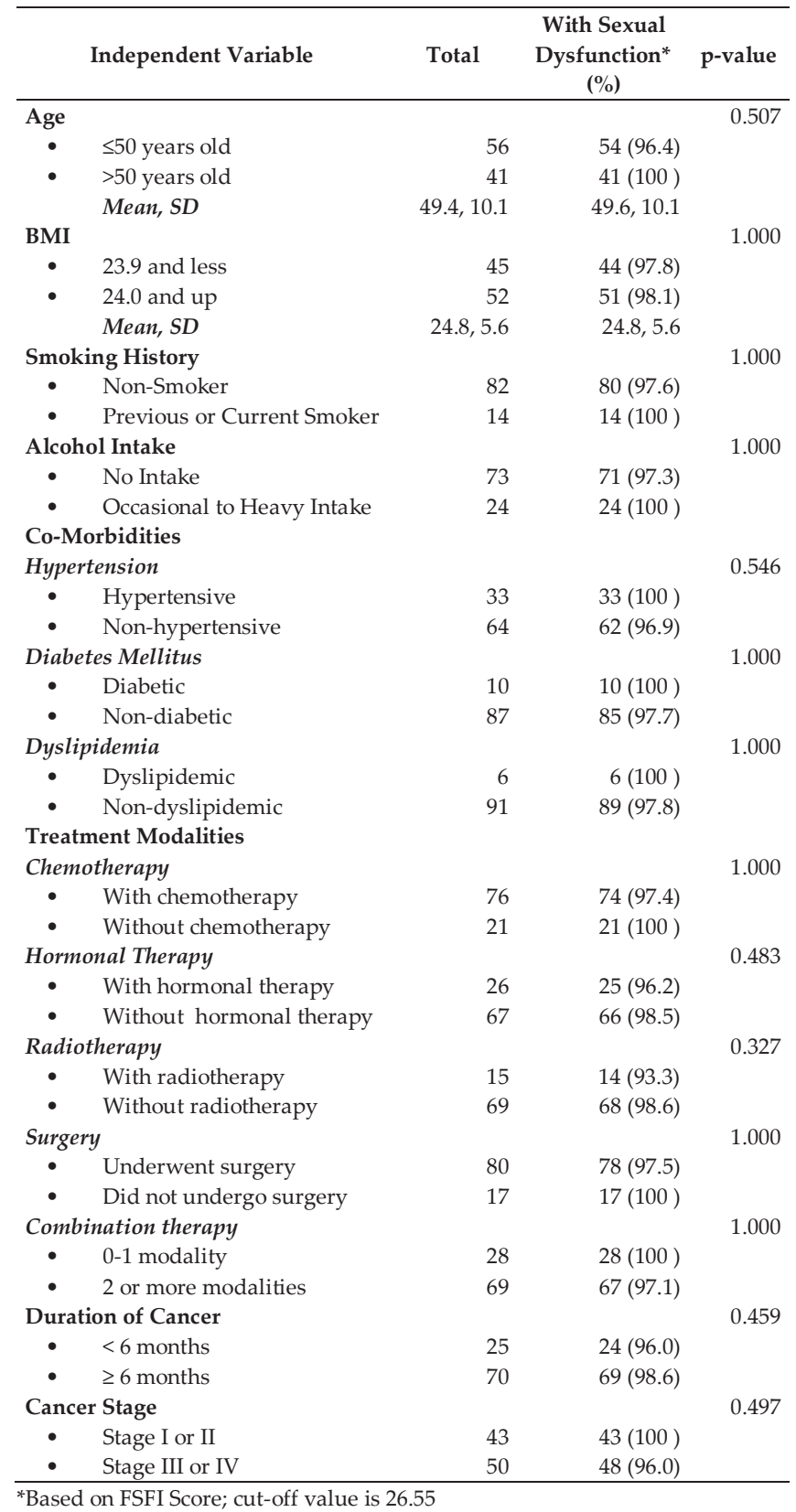

\section{Discussion}

Sexuality and sexual experience are important domains of human experience that could have a devastating turnaround for breast cancer patients upon cancer diagnosis and in the physical and psychological aftermath. Sexual dysfunction is an aspect in the health-related quality of life, but may be lacking adequate attention by healthcare providers, during and following cancer treatment.

Results of our study showed that $97.9 \%(n=95 / 97)$ of the respondents experience sexual dysfunction, which is similar to prevalence rates $(64-98 \%)$ in other studies, ${ }^{3-5,7}$ noting relative similarity across cultures (Netherlands, Australia, France, Italy, Japan, Philippines).

This very high prevalence rate tells us that sexual dysfunction among Filipina breast cancer patients is a frequent occurrence and is one aspect that should not be ignored in the management of the disease.

No significant relationship was seen between sexual dysfunction and the factors considered in this study, which indicates that regardless of patient, treatment, and disease profile of the Filipina breast cancer patient, sexual function is disturbed and thus all such patients should be managed accordingly. It is important that the physicians discuss this issue with their patients.

Oncologists and other healthcare providers often feel reluctant to raise the subject for several reasons, possibly because of inadequate training in discussing sexual matters, personal or patient awkwardness or lack of time, especially for institutions with a high volume of patients such as government hospitals. This leaves the patients uninformed, not meeting their needs and affecting their daily lives. They may think that sexual dysfunction is a treatment side effect to which there are no solutions and that they just have to endure. Including sexual health as a part of the routine oncologic treatment plan and follow up should be included in all practices providing breast cancer treatment.

Practicing a multidisciplinary approach, involving also sex therapists and pelvic physical therapists, contributes to the management of associated conditions that might be contributory to the problem. Counseling, patient support groups and involving the sexual partners in the management plan and follow up might also yield positive results.

In conclusion, there is a very high prevalence rate of sexual dysfunction among Filipinas with breast cancer, thus, sexual health as part of the routine oncologic treatment plan and follow up should be included in all practices providing quality breast cancer treatment. 


\section{References}

1. Philippine Cancer Society Inc. 2010 Philippine Cancer facts and Estimates. Manila: PCSI, 2010.

2. Barni S, Mondin R. Sexual dysfunction in treated breast cancer patients. Ann Oncol. 1997; 8(2):149-53.

3. Brédart A, Dolbeault S, Savignoni A, et al. Prevalence and associated factors of sexual problems after early-stage breast cancer treatment: results of a French exploratory survey. Psychooncology. 2011; 20(8):84150 .

4. Kedde H, van de Wiel HB, Weijmar Schultz WC, Wijsen C. Sexual dysfunction in young women with breast cancer. Support Care Cancer. 2013; 21(1):271-80.

5. Panjari M, Bell RJ, Davis SR. Sexual function after breast cancer. J Sex Med. 2011; 8(1):294-302.

6. Kaplan BJ, Sadock VA. Synopsis of Psychiatry: Behavioral Sciences and Clinical Psychiatry, $10^{\text {th }}$ ed. Baltimore: Lippincott Williams and Wilkins, 2007.

7. Takahashi M, Ohno S, Inoue H, et al. Impact of breast cancer diagnosis and treatment on women's sexuality: a survey of Japanese patients. Psychooncology. 2008; 17(9):901-7.
8. Cavalheiro JA, Bittelbrunn A, Menke $\mathrm{CH}$, et al. Sexual function and chemotherapy in postmenopausal women with breast cancer. BMC Womens Health. 2012; 12:28.

9. Sbitti Y, Kadiri H, Essaidi I, et al. Breast cancer treatment and sexual dysfunction: Moroccan women's perception. BMC Womens Health. 2011; 11:29.

10. National Breast Cancer Centre and the National Cancer Control Initiative. Clinical practice guidelines for the psychosocial care of adults with cancer. NHMRC: Australia, 2003.

11. Thors CL, Broeckel JA, Jacobsen PB. Sexual functioning in breast cancer survivors. Cancer Control. 2001; 8(5):442-8.

12. Rillon-Tabil N, Malong C, Vicera J, Gomez H. Translation and validity of the Female Sexual Function Index Filipino Version (FSFI-Fil). Philipp J Intern Med. 2013; 51(4):1-11.

13. Lui A, et al. Prevalence of sexual dysfunction among women with diabetes at The UP-PGH Outpatient Department. Manila, 2011. (Unpublished)

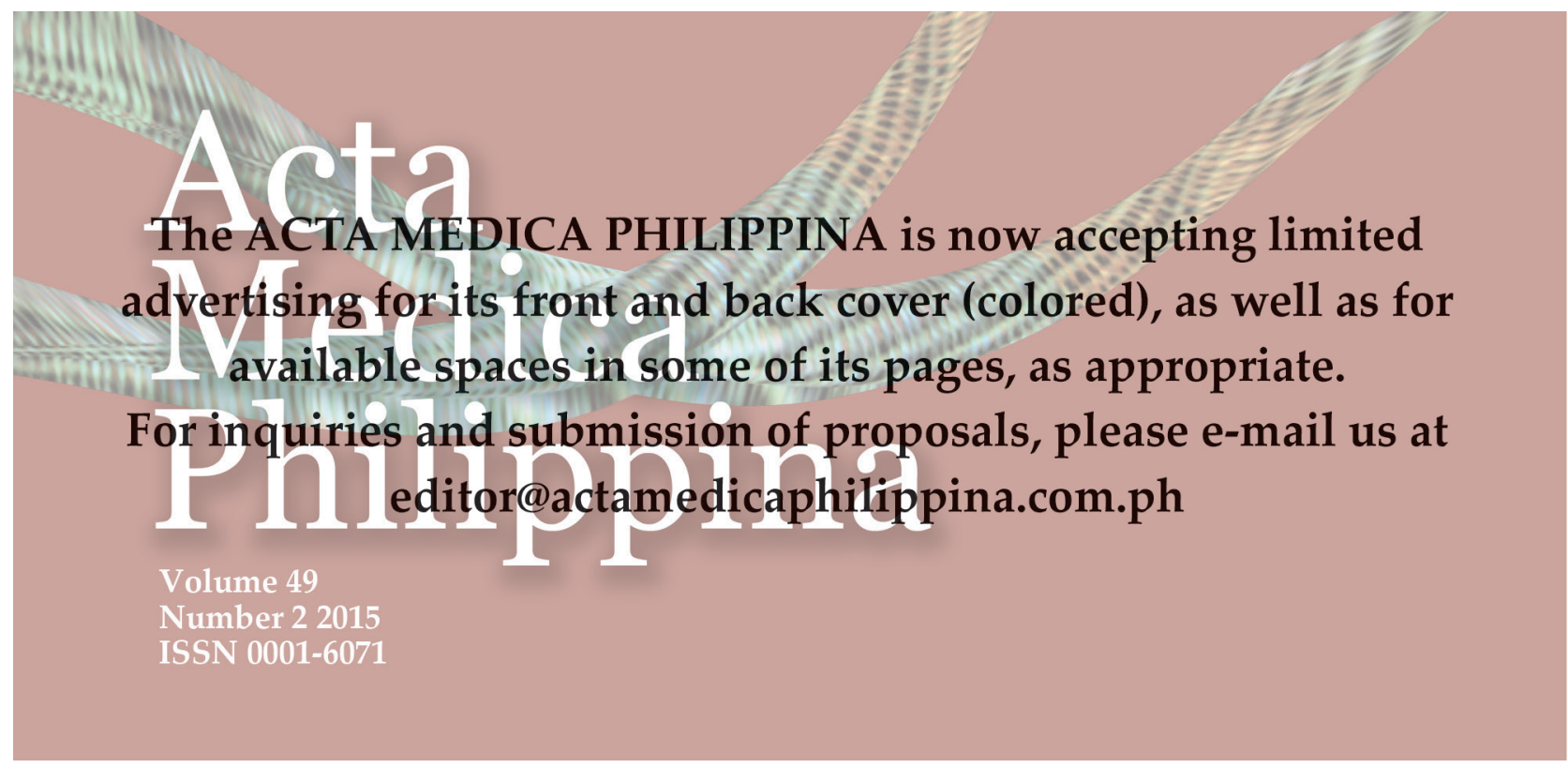

